\title{
Computing cup products in integral cohomology of Hilbert schemes of points on K3 surfaces
}

\author{
Simon Kapfer
}

\begin{abstract}
We study cup products in the integral cohomology of the Hilbert scheme of $n$ points on a K3 surface and present a computer program for this purpose. In particular, we deal with the question of which classes can be represented by products of lower degrees.

Supplementary materials are available with this article.
\end{abstract}

\section{Introduction}

The Hilbert schemes of $n$ points on a complex surface parametrise all zero-dimensional subschemes of length $n$. Studying their rational cohomology, Nakajima [10] was able to give an explicit description of the vector space structure in terms of the action of a Heisenberg algebra. The Hilbert schemes of points on a K3 surface are one of the few known classes of irreducible holomorphic symplectic manifolds. Lehn and Sorger [6] developed an algebraic model to describe the cohomological ring structure. On the other hand, Qin and Wang [11] found a base for integral cohomology in the projective case. By combining these results, we are able to compute all cup products in the cohomology rings of Hilbert schemes of $n$ points on a projective K3 surface with integral coefficients. For $n=2$, this was done by Boissière et al. [1], who applied their results to automorphism groups of prime order. When $n$ is increasing, the ranks of the cohomology rings become very large, so we need the help of a computer. The source code is available from https://github.com/s--kapfer/HilbK3 and is also available as online supplementary material from the publisher's website.

Our goal here is to give some properties for low degrees. Denote by $S^{[3]}$ the Hilbert scheme of three points on a projective K3 surface (or a deformation equivalent space). We identify $\operatorname{Sym}^{k} H^{2}\left(S^{[n]}, \mathbb{Z}\right)$ with its image in $H^{2 k}\left(S^{[n]}, \mathbb{Z}\right)$ under the cup product mapping.

TheOREM 0.1. The cup product mappings for the Hilbert scheme of three points on a projective K3 surface have cokernels

$$
\begin{gathered}
\frac{H^{4}\left(S^{[3]}, \mathbb{Z}\right)}{\operatorname{Sym}^{2} H^{2}\left(S^{[3]}, \mathbb{Z}\right)} \cong \frac{\mathbb{Z}}{3 \mathbb{Z}} \oplus \mathbb{Z}^{\oplus 23}, \\
\frac{H^{6}\left(S^{[3]}, \mathbb{Z}\right)}{H^{2}\left(S^{[3]}, \mathbb{Z}\right) \smile H^{4}\left(S^{[3]}, \mathbb{Z}\right)} \cong\left(\frac{\mathbb{Z}}{3 \mathbb{Z}}\right)^{\oplus 23} .
\end{gathered}
$$

Although the case $n=3$ is the most interesting for us, our computer program allows computations for arbitrary $n$. We give some numerical results in $\S 2$.

Received 25 February 2015; revised 6 October 2015.

2010 Mathematics Subject Classification 14J10, 14Q15 (primary), 05A17, 55N32 (secondary). 


\section{Preliminaries}

Definition 1.1. Let $n$ be a natural number. A partition of $n$ is a decreasing sequence $\lambda=\left(\lambda_{1}, \ldots, \lambda_{k}\right), \lambda_{1} \geqslant \ldots \geqslant \lambda_{k}>0$ of natural numbers such that $\sum_{i} \lambda_{i}=n$. Sometimes it is convenient to write $\lambda=\left(\ldots, 2^{m_{2}}, 1^{m_{1}}\right)$ with multiplicities in the exponent. No confusion should be possible since numerical exponentiation is never meant in this context. We define the weight $\|\lambda\|:=\sum m_{i} i=n$ and the length $|\lambda|:=\sum_{i} m_{i}=k$. We also define $z_{\lambda}:=\prod_{i} i^{m_{i}} m_{i} !$.

Definition 1.2. Let $\Lambda_{n}:=\mathbb{Q}\left[x_{1}, \ldots, x_{n}\right]^{S_{n}}$ be the graded ring of symmetric polynomials. There are canonical projections : $\Lambda_{n+1} \rightarrow \Lambda_{n}$ which send $x_{n+1}$ to zero. The graded projective $\operatorname{limit} \Lambda:=\lim _{\leftarrow} \Lambda_{n}$ is called the ring of symmetric functions. Let $m_{\lambda}$ and $p_{\lambda}$ denote the monomial and the power sum symmetric functions, respectively. They are defined as follows. For a monomial $x_{i_{1}}^{\lambda_{1}} x_{i_{2}}^{\lambda_{2}} \ldots x_{i_{k}}^{\lambda_{k}}$ of total degree $n$, the (ordered) sequence of exponents $\left(\lambda_{1}, \ldots, \lambda_{k}\right)$ defines a partition $\lambda$ of $n$, which is called the shape of the monomial. Then we define $m_{\lambda}$ as the sum of all monomials of shape $\lambda$. For the power sums, first define $p_{n}:=x_{1}^{n}+x_{2}^{n}+\ldots$. Then $p_{\lambda}:=p_{\lambda_{1}} p_{\lambda_{2}} \ldots p_{\lambda_{k}}$.

The families $\left(m_{\lambda}\right)_{\lambda}$ and $\left(p_{\lambda}\right)_{\lambda}$ form two $\mathbb{Q}$-bases of $\Lambda$, so they are linearly related by $p_{\lambda}=$ $\sum_{\mu} \psi_{\lambda \mu} m_{\mu}$. It turns out that the base change matrix $\left(\psi_{\lambda \mu}\right)$ has integral entries, but its inverse $\left(\psi_{\mu \lambda}^{-1}\right)$ has not. A method to determine the $\left(\psi_{\lambda \mu}\right)$ is given by Lascoux $[\mathbf{5}, \S 3.7]$.

Definition 1.3. A lattice $L$ is a free $\mathbb{Z}$-module of finite rank, equipped with a nondegenerate symmetric integral bilinear form $B$. The lattice $L$ is called odd if there exists a $v \in L$ such that $B(v, v)$ is odd; otherwise it is called even. If the map $v \mapsto B(v, v)$ takes both negative and positive values on $L$, the lattice is called indefinite. Choosing a base $\left\{e_{i}\right\}_{i}$ of our lattice, we can write $B$ as a symmetric matrix. $L$ is called unimodular, if the matrix $B$ has determinant \pm 1 . The difference between the number of positive eigenvalues and the number of negative eigenvalues of $B$ (regarded as a matrix over $\mathbb{R}$ ) is called the signature.

There is the following classification theorem (see [9, Chapter II]).

Theorem 1.4. Two indefinite unimodular lattices $L, L^{\prime}$ are isometric if and only if they have the same rank, signature and parity. Evenness implies that the signature is divisible by eight. In particular, if $L$ is odd, then $L$ possesses an orthogonal basis and is hence isometric to $\langle 1\rangle^{\oplus k} \oplus\langle-1\rangle^{\oplus l}$ for some $k, l \geqslant 0$. If $L$ is even, then $L$ is isometric to $U^{\oplus k} \oplus\left( \pm E_{8}\right)^{\oplus l}$ for some $k, l \geqslant 0$.

Definition 1.5. Let $S$ be a projective K3 surface. We fix integral bases 1 of $H^{0}(S, \mathbb{Z}), x$ of $H^{4}(S, \mathbb{Z})$ and $\alpha_{1}, \ldots, \alpha_{22}$ of $H^{2}(S, \mathbb{Z})$. The cup product induces a symmetric bilinear form $B_{H^{2}}$ on $H^{2}(S, \mathbb{Z})$ and thus the structure of a unimodular lattice. We may extend $B_{H^{2}}$ to a symmetric non-degenerate bilinear form $B$ on $H^{*}(S, \mathbb{Z})$ by setting $B(1,1)=0, B\left(1, \alpha_{i}\right)=$ $0, B(1, x)=1, B(x, x)=0$.

By the Hirzebruch index theorem, we know that $H^{2}(S, \mathbb{Z})$ has signature -16 and, by the classification theorem for indefinite unimodular lattices, is isomorphic to $U^{\oplus 3} \oplus\left(-E_{8}\right)^{\oplus 2}$.

Definition 1.6. $B$ induces a form $B \otimes B$ on $\operatorname{Sym}^{2} H^{*}(S, \mathbb{Z})$. So the cup product

$$
\mu: \operatorname{Sym}^{2} H^{*}(S, \mathbb{Z}) \longrightarrow H^{*}(S, \mathbb{Z})
$$

induces an adjoint comultiplication $\Delta$ that is coassociative, given by

$$
\Delta: H^{*}(S, \mathbb{Z}) \longrightarrow \operatorname{Sym}^{2} H^{*}(S, \mathbb{Z}), \quad \Delta=-(B \otimes B)^{-1} \mu^{T} B,
$$


with the property $(B \otimes B)(\Delta(a), b \otimes c)=-B(a, b \smile c)$. Note that this does not define a bialgebra structure. The image of 1 under the composite map $\mu \circ \Delta$, denoted by $e=24 x$ is called the Euler Class.

More generally, every linear map $f: A^{\otimes k} \rightarrow A^{\otimes m}$ induces an adjoint map $g$ in the other direction that satisfies $(-1)^{m} B^{\otimes m}(f(x), y)=(-1)^{k} B^{\otimes k}(x, g(y))$.

We denote by $S^{[n]}$ the Hilbert scheme of $n$ points on $S$ : that is, the classifying space of all zero-dimensional closed subschemes of length $n . S^{[0]}$ consists of a single point and $S^{[1]}=S$. Fogarty [4, Theorem 2.4] proved that the Hilbert scheme is a smooth variety. A theorem by Nakajima [10] gives an explicit description of the vector space structure of $H^{*}\left(S^{[n]}, \mathbb{Q}\right)$ in terms of creation operators

$$
\mathfrak{q}_{l}(\beta): H^{*}\left(S^{[n]}, \mathbb{Q}\right) \longrightarrow H^{*+k+2(l-1)}\left(S^{[n+l]}, \mathbb{Q}\right),
$$

where $\beta \in H^{k}(S, \mathbb{Q})$, acting on the direct sum $\mathbb{H}:=\bigoplus_{n} H^{*}\left(S^{[n]}, \mathbb{Q}\right)$. The operators $\mathfrak{q}_{l}(\beta)$ are linear and commute with each other. The vacuum vector $|0\rangle$ is defined as the generator of $H^{0}\left(S^{[0]}, \mathbb{Q}\right) \cong \mathbb{Q}$. The images of $|0\rangle$ under the polynomial algebra generated by the creation operators span $\mathbb{H}$ as a vector space. Following $[\mathbf{1 1}]$, we abbreviate $\mathfrak{q}_{l_{1}}(\beta) \ldots \mathfrak{q}_{l_{k}}(\beta)=: \mathfrak{q}_{\lambda}(\beta)$, where the partition $\lambda$ is composed by the $l_{i}$.

An integral basis for $H^{*}\left(S^{[n]}, \mathbb{Z}\right)$ in terms of Nakajima's operators was given by Qin-Wang.

TheOrem 1.7 [11, Theorem 5.4]. Let $\mathfrak{m}_{\nu, \alpha}:=\sum_{\rho} \psi_{\nu \rho}^{-1} \mathfrak{q}_{\rho}(\alpha)$, with coefficients $\psi_{\nu \rho}^{-1}$, as in Definition 1.2. The classes

$$
\frac{1}{z_{\lambda}} \mathfrak{q}_{\lambda}(1) \mathfrak{q}_{\mu}(x) \mathfrak{m}_{\nu^{1}, \alpha_{1}} \ldots \mathfrak{m}_{\nu^{22}, \alpha_{22}}|0\rangle, \quad\|\lambda\|+\|\mu\|+\sum_{i=1}^{22}\left\|\nu^{i}\right\|=n
$$

form an integral basis for $H^{*}\left(S^{[n]}, \mathbb{Z}\right)$. Here, $\lambda, \mu, \nu^{i}$ are partitions.

Notation 1.8. To enumerate the basis of $H^{*}\left(S^{[n]}, \mathbb{Z}\right)$, we introduce the abbreviation

$$
\boldsymbol{\alpha}^{\boldsymbol{\lambda}}:=1^{\lambda^{0}} \alpha_{1}^{\lambda^{1}} \ldots \alpha_{22}^{\lambda^{22}} x^{\lambda^{23}}:=\frac{1}{z_{\widetilde{\lambda^{0}}}} \mathfrak{q}_{\lambda^{0}}(1) \mathfrak{q}_{\lambda^{23}}(x) \mathfrak{m}_{\lambda^{1}, \alpha_{1}} \ldots \mathfrak{m}_{\lambda^{22}, \alpha_{22}}|0\rangle
$$

where the partition $\widetilde{\lambda^{0}}$ is built from $\lambda^{0}$ by appending a sufficient number of ones such that $\left\|\widetilde{\lambda^{0}}\right\|+\sum_{i \geqslant 1}\left\|\lambda^{i}\right\|=n$. If $\sum_{i \geqslant 0}\left\|\lambda^{i}\right\|>n$, we put $\boldsymbol{\alpha}^{\boldsymbol{\lambda}}=0$. Thus we can interpret $\boldsymbol{\alpha}^{\boldsymbol{\lambda}}$ as an element of $H^{*}\left(S^{[n]}, \mathbb{Z}\right)$ for arbitrary $n$. We say that the symbol $\boldsymbol{\alpha}^{\boldsymbol{\lambda}}$ is reduced, if $\lambda^{0}$ contains no ones. We also define $\|\boldsymbol{\lambda}\|:=\sum_{i \geqslant 0}\left\|\lambda^{i}\right\|$.

Lemma 1.9. Let $\boldsymbol{\alpha}^{\boldsymbol{\lambda}}$ represent a class of cohomological degree $2 k$. If $\boldsymbol{\alpha}^{\boldsymbol{\lambda}}$ is reduced, then $k / 2 \leqslant\|\boldsymbol{\lambda}\| \leqslant 2 k$.

Proof. This is a simple combinatorial observation. We give the two extremal cases. The lowest ratio between $\|\boldsymbol{\lambda}\|$ and $\operatorname{deg} \boldsymbol{\alpha}^{\boldsymbol{\lambda}}$ is achieved by the classes $x^{\left(1^{m}\right)}$, where the degree is $4 m$ and the weight of $\boldsymbol{\lambda}$ is $m$. The highest ratio is achieved by the classes $1^{\left(2^{m}\right)}$, where both degree and weight equal $2 m$. So $1 / 4 \leqslant\|\boldsymbol{\lambda}\| /\left(\operatorname{deg} \boldsymbol{\alpha}^{\boldsymbol{\lambda}}\right) \leqslant 1$.

The ring structure of $H^{*}\left(S^{[n]}, \mathbb{Q}\right)$ has been studied by Lehn and Sorger in [6], where an explicit algebraic model is constructed. We recall it briefly here.

DeFINITION $1.10[6, \S 2]$. Let $\pi$ be a permutation of $n$ letters, written as a product of disjoint cycles. To each cycle we may associate an element of $A:=H^{*}(S, \mathbb{Q})$. This defines an element in $A^{\otimes m}, m$ being the number of cycles. For example, a term such as $(123)_{\alpha_{1}}(45)_{\alpha_{2}}$ may describe 
a permutation consisting of two cycles with associated classes $\alpha_{1}, \alpha_{2} \in A$. We interpret the cycles as the orbits of the subgroup $\langle\pi\rangle \subset S_{n}$ generated by $\pi$. We denote the set of orbits by $\langle\pi\rangle \backslash[n]$. Thus we construct a vector space $A\left\{S_{n}\right\}:=\bigoplus_{\pi \in S_{n}} A^{\otimes\langle\pi\rangle \backslash[n]}$.

To define a ring structure, take two permutations $\pi, \tau \in S_{n}$ and the subgroup $\langle\pi, \tau\rangle$ generated by them. The natural map of orbit spaces $p_{\pi}:\langle\pi\rangle \backslash[n] \rightarrow\langle\pi, \tau\rangle \backslash[n]$ induces a map $f^{\pi,\langle\pi, \tau\rangle}: A^{\otimes\langle\pi\rangle \backslash[n]} \rightarrow A^{\otimes\langle\pi, \tau\rangle \backslash[n]}$, which multiplies the factors of an elementary tensor if the corresponding orbits are glued together. Denote by $f_{\langle\pi, \tau\rangle, \pi}$ the adjoint to this map in the sense of Definition 1.6. Then the map

$$
\begin{gathered}
m_{\pi, \tau}: A^{\otimes\langle\pi\rangle \backslash[n]} \otimes A^{\otimes\langle\tau\rangle \backslash[n]} \longrightarrow A^{\otimes\langle\pi \tau\rangle \backslash[n]}, \\
a \otimes b \longmapsto f_{\langle\pi, \tau\rangle, \pi \tau}\left(f^{\pi,\langle\pi, \tau\rangle}(a) \cdot f^{\tau,\langle\pi, \tau\rangle}(b) \cdot e^{g(\pi, \tau)}\right)
\end{gathered}
$$

defines a multiplication on $A\left\{S_{n}\right\}$. Here the dot means the cup product on each tensor factor and $e^{g(\pi, \tau)} \in A^{\otimes\langle\pi, \tau\rangle \backslash[n]}$ is an elementary tensor that is composed of powers of the Euler class $e$ : for each orbit $B \in \otimes\langle\pi, \tau\rangle \backslash[n]$ the exponent $g(\pi, \tau)(B)$ (so-called 'graph defect'; see [6, 2.6]) is given by

$$
g(\pi, \tau)(B)=\frac{1}{2}\left(|B|+2-\left|p_{\pi}^{-1}(\{B\})\right|-\left|p_{\tau}^{-1}(\{B\})\right|-\left|p_{\pi \tau}^{-1}(\{B\})\right|\right) .
$$

The symmetric group $S_{n}$ acts on $A\left\{S_{n}\right\}$ by conjugation, permuting the direct summands:

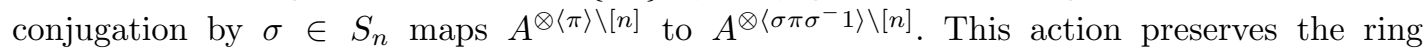
structure. Therefore the space of invariants $A^{[n]}:=\left(A\left\{S_{n}\right\}\right)^{S_{n}}$ becomes a subring. The main theorem of $[\mathbf{6}]$ can now be stated.

Theorem 1.11 [6, Theorem 3.2]. The following map is an isomorphism of rings:

$$
\begin{aligned}
H^{*}\left(S^{[n]}, \mathbb{Q}\right) & \longrightarrow A^{[n]}, \\
\mathfrak{q}_{n_{1}}\left(\beta_{1}\right) \ldots \mathfrak{q}_{n_{k}}\left(\beta_{k}\right)|0\rangle & \longmapsto \sum_{\sigma \in S_{n}} \sigma a \sigma^{-1},
\end{aligned}
$$

with $\sum_{i} n_{i}=n$ and $a=\left(12 \ldots n_{1}\right)_{\beta_{1}}\left(n_{1}+1 \ldots n_{1}+n_{2}\right)_{\beta_{2}} \ldots\left(n-n_{k} \ldots n\right)_{\beta_{k}} \in A\left\{S_{n}\right\}$.

Since $H^{\text {odd }}\left(S^{[n]}, \mathbb{Z}\right)=0$ and $H^{\text {even }}\left(S^{[n]}, \mathbb{Z}\right)$ is torsion-free by [7], we can apply these results to $H^{*}\left(S^{[n]}, \mathbb{Z}\right)$ to determine the multiplicative structure of cohomology with integer coefficients. It turns out that it is somehow independent of $n$. More precisely, we have the following stability theorem, by Li, Qin and Wang.

Theorem 1.12 (Derived from [11, Theorem 2.1]). Let $Q_{1}, \ldots, Q_{s}$ be products of creation operators: that is $Q_{i}=\prod_{j} \mathfrak{q}_{\lambda_{i, j}}\left(\beta_{i, j}\right)$ for some partitions $\lambda_{i, j}$ and classes $\beta_{i, j} \in H^{*}(S, \mathbb{Z})$. Set $n_{i}:=\sum_{j}\left\|\lambda_{i, j}\right\|$. Then the cup product $\prod_{i=1}^{s}\left(\left(1 /\left(n-n_{i}\right) !\right) \mathfrak{q}_{1^{n-n_{i}}}(1) Q_{i}|0\rangle\right)$ is equal to a finite linear combination of classes of the form $(1 /(n-m) !) \mathfrak{q}_{1^{n-m}}(1) \prod_{j} \mathfrak{q}_{\mu_{j}}\left(\gamma_{j}\right)|0\rangle$, with $\gamma \in H^{*}(S, \mathbb{Z}), m=\sum_{j}\left\|\mu_{j}\right\|$, whose coefficients are independent of $n$. We have the upper bound $m \leqslant \sum_{i} n_{i}$. Moreover, $m=\sum_{i} n_{i}$ if and only if the corresponding class is $(1 /(n-m) !) \mathfrak{q}_{1^{n-m}}(1) Q_{1} Q_{2} \ldots Q_{s}|0\rangle$ with coefficient one.

Corollary 1.13. Let $\boldsymbol{\alpha}^{\boldsymbol{\lambda}}, \boldsymbol{\alpha}^{\mu}, \boldsymbol{\alpha}^{\boldsymbol{\nu}}$ be reduced. Assume $n \geqslant\|\boldsymbol{\lambda}\|,\|\boldsymbol{\mu}\|$. Then the coefficients $c_{\boldsymbol{\nu}}^{\boldsymbol{\lambda} \mu}$ of the cup product in $H^{*}\left(S^{[n]}, \mathbb{Z}\right)$

$$
\alpha^{\lambda} \smile \alpha^{\mu}=\sum_{\nu} c_{\nu}^{\lambda \mu} \alpha^{\nu}
$$

are polynomials in $n$ of degree at most $\|\boldsymbol{\lambda}\|+\|\boldsymbol{\mu}\|-\|\boldsymbol{\nu}\|$. 
Proof. Set $Q_{\boldsymbol{\lambda}}:=\mathfrak{q}_{\lambda^{0}}(1) \mathfrak{q}_{\lambda^{23}}(x) \prod_{1 \leqslant j \leqslant 22} \mathfrak{q}_{\lambda^{j}}\left(\alpha_{j}\right)$ and $n_{\boldsymbol{\lambda}}:=\|\boldsymbol{\lambda}\|$. Then $\boldsymbol{\alpha}^{\boldsymbol{\lambda}}=$ $\left(1 /\left(\left(n-n_{\boldsymbol{\lambda}}\right) ! z_{\lambda^{0}}\right)\right) \mathfrak{q}_{1^{n-n_{\boldsymbol{\lambda}}}}(1) Q_{\boldsymbol{\lambda}}|0\rangle$ and $\boldsymbol{\alpha}^{\boldsymbol{\mu}}=\left(1 /\left(\left(n-n_{\boldsymbol{\mu}}\right) ! z_{\mu^{0}}\right)\right) \mathfrak{q}_{1^{n-n_{\boldsymbol{\mu}}}}(1) Q_{\boldsymbol{\mu}}|0\rangle$. Thus the coefficient $c_{\boldsymbol{\nu}}^{\boldsymbol{\lambda} \boldsymbol{\mu}}$ in the product expansion is a constant, which depends on $\|\boldsymbol{\lambda}\|,\|\boldsymbol{\mu}\|,\|\boldsymbol{\nu}\|$, but not on $n$, multiplied by $\left(n-n_{\boldsymbol{\nu}}\right) ! /(n-m)$ ! for a certain $m \leqslant n_{\boldsymbol{\lambda}}+n_{\boldsymbol{\mu}}$. This is a polynomial of degree $m-n_{\boldsymbol{\nu}} \leqslant n_{\boldsymbol{\lambda}}+n_{\boldsymbol{\mu}}-n_{\boldsymbol{\nu}}=\|\boldsymbol{\lambda}\|+\|\boldsymbol{\mu}\|-\|\boldsymbol{\nu}\|$.

REMARK 1.14. If $n<\|\boldsymbol{\lambda}\|$ or $n<\|\boldsymbol{\mu}\|$, one has $\boldsymbol{\alpha}^{\boldsymbol{\lambda}}=0$ (respectively, $\boldsymbol{\alpha}^{\boldsymbol{\mu}}=0$ ). But it is still possible that $\boldsymbol{\alpha}^{\boldsymbol{\nu}} \neq 0$ in $H^{*}\left(S^{[n]}\right)$. It seems that, in this case, the polynomial $c_{\boldsymbol{\nu}}^{\boldsymbol{\lambda} \mu}$ always becomes zero when evaluated at $n$. So the $c_{\boldsymbol{\nu}}^{\boldsymbol{\lambda} \mu}$ seem to be universal in the sense that the above corollary holds true even without the condition $n \geqslant\|\boldsymbol{\lambda}\|,\|\boldsymbol{\mu}\|$.

EXAmPLE 1.15. Here are some explicit examples for illustration. See A.1 for how to compute them.

(1) $1^{(2,2)} \smile \alpha_{i}^{(2)}=-2 \cdot 1^{(2)} \alpha_{i}^{(1)} x^{(1)}+1^{(2,2)} \alpha_{i}^{(2)}+2 \cdot 1^{(2)} \alpha_{i}^{(3)}+\alpha_{i}^{(4)}$ for $i \in\{1 . .22\}$.

(2) Let $i, j \in\{1 \ldots 22\}$. If $i \neq j$, then $\alpha_{i}^{(2)} \smile \alpha_{j}^{(1)}=\alpha_{i}^{(2)} \alpha_{j}^{(1)}+2 B\left(\alpha_{i}, \alpha_{j}\right) \cdot x^{(1)}$. Otherwise, $\alpha_{i}^{(2)} \smile \alpha_{i}^{(1)}=\alpha_{i}^{(3)}+\alpha_{i}^{(2,1)}+2 B\left(\alpha_{i}, \alpha_{i}\right) \cdot x^{(1)}$.

(3) Set $\boldsymbol{\alpha}^{\boldsymbol{\lambda}}=1^{(2)}$ and $\boldsymbol{\alpha}^{\boldsymbol{\nu}}=x^{(1)}$. Then $c_{\boldsymbol{\nu}}^{\boldsymbol{\lambda} \boldsymbol{\lambda}}=-(n-1)$.

(4) Set $\boldsymbol{\alpha}^{\boldsymbol{\lambda}}=1^{(2,2)}$ and $\boldsymbol{\alpha}^{\boldsymbol{\nu}}=x^{(1,1)}$. Then $c_{\boldsymbol{\nu}}^{\boldsymbol{\lambda} \boldsymbol{\lambda}}=(n-3)(n-2) / 2$.

EXAMPLE 1.16. Let $i, j$ be indices, such that $B\left(\alpha_{i}, \alpha_{j}\right)=1, B\left(\alpha_{i}, \alpha_{i}\right)=0=B\left(\alpha_{j}, \alpha_{j}\right)$ and let $k \geqslant 0$. Set $\boldsymbol{\alpha}^{\boldsymbol{\lambda}}=\alpha_{i}^{(1)} \alpha_{j}^{(1)} x^{\left(1^{k}\right)}$ and $\boldsymbol{\alpha}^{\boldsymbol{\nu}}=x^{\left(1^{2 k+2}\right)}$. Then $c_{\boldsymbol{\nu}}^{\boldsymbol{\lambda} \boldsymbol{\lambda}}=1$.

Proof. It is not hard to see, from the definition, that, for $\beta_{j}, \gamma_{j} \in H^{*}(S)$,

$$
\mathfrak{q}_{1}\left(\beta_{1}\right) \ldots \mathfrak{q}_{1}\left(\beta_{n}\right)|0\rangle \smile \mathfrak{q}_{1}\left(\gamma_{1}\right) \ldots \mathfrak{q}_{1}\left(\gamma_{n}\right)|0\rangle=\sum_{\sigma \in S_{n}} \mathfrak{q}_{1}\left(\beta_{1} \cdot \gamma_{\sigma(1)}\right) \ldots \mathfrak{q}_{1}\left(\beta_{n} \cdot \gamma_{\sigma(n)}\right)|0\rangle .
$$

A combinatorial investigation yields

$$
\left(\mathfrak{q}_{1}\left(\alpha_{i}\right) \mathfrak{q}_{1}\left(\alpha_{j}\right) \mathfrak{q}_{1}(x)^{k} \mathfrak{q}_{1}(1)^{k+m}|0\rangle\right)^{2}=\frac{(k+m) !^{2}}{m !} \mathfrak{q}_{1}(x)^{2 k+2} \mathfrak{q}_{1}(1)^{m}|0\rangle+\text { other terms }
$$

Looking at 1.8 , the result follows.

Theorem 1.17. The quotient

$$
\frac{H^{2 k}\left(S^{[n]}, \mathbb{Z}\right)}{\operatorname{Sym}^{k} H^{2}\left(S^{[n]}, \mathbb{Z}\right)}
$$

is a free $\mathbb{Z}$-module for $n \geqslant k+2$.

Proof. The idea of the proof is to modify the basis of $H^{2 k}\left(S^{[n]}, \mathbb{Z}\right)$, given in Theorem 1.7, in such a way that $\operatorname{Sym}^{k} H^{2}\left(S^{[n]}, \mathbb{Z}\right)$ splits as a direct summand.

Given a free $\mathbb{Z}$-module $M$ with basis $\left(b_{i}\right)_{i=1, \ldots, m}$ and a vector $v=a_{1} b_{1}+\ldots+a_{m} b_{m}$, there is another basis of $M$ which contains $v$ if and only if $\operatorname{gcd}\left\{a_{1}, \ldots, a_{m}\right\}=1$. More generally, given a set of vectors $\left(v_{i}\right)_{i=1, \ldots, r}, v_{i}=a_{i 1} b_{1}+\ldots+a_{i m} b_{m}$, we can complete it to a basis of $M$ if and only if the $r \times r$-minors of the matrix $\left(a_{i j}\right)_{i j}$ share no common divisor. We want to show that the canonical basis of $\operatorname{Sym}^{k} H^{2}\left(S^{[n]}, \mathbb{Z}\right)$ is such a set.

A basis of $H^{2}\left(S^{[n]}, \mathbb{Z}\right)$ is given by the classes $\alpha_{i}^{(1)}=(1 /(n-1) !) \mathfrak{q}_{1^{n-1}}(1) \mathfrak{q}_{1}\left(\alpha_{i}\right)|0\rangle, i=$ $1, \ldots, 22$ and $1^{(2)}=(1 /(2(n-2) !)) \mathfrak{q}_{\left(2,1^{n-2}\right)}(1)|0\rangle$. A power of $\alpha_{i}^{(1)}$ looks like (Theorem 1.12)

$$
\left(\alpha_{i}^{(1)}\right)^{k}=\frac{1}{(n-k) !} \mathfrak{q}_{1^{n-k}}(1) \mathfrak{q}_{1^{k}}\left(\alpha_{i}\right)|0\rangle+\text { other terms containing } \mathfrak{q}_{\lambda}(x) .
$$


Now, by the definition of $\psi_{\lambda \mu}, \mathfrak{q}_{1^{k}}\left(\alpha_{i}\right)=\mathfrak{m}_{(k), \alpha_{i}}+\ldots+k ! \cdot \mathfrak{m}_{\left(1^{k}\right), \alpha_{i}}$, so

$$
\left(\alpha_{i}^{(1)}\right)^{k}=\alpha_{i}^{(k)}+\text { other terms. }
$$

Next, we determine the coefficients of $1^{(k+1)}$ and $1^{(k, 2)}$ in the expansion of $\left(1^{(2)}\right)^{k}$. Considering Definition 1.10, we observe that here the graph defect is zero and the adjoint map is trivial, so the problem reduces to combinatorics of the symmetric group: the coefficient of $1^{(k+1)}$ is the number of ways of writing a $(k+1)$-cycle as a product of $k$ transpositions. A result of Dénes [2] states that this is $(k+1)^{k-1}$. For the $1^{(k, 2)}$-coefficient, we have to choose one transposition and write a $k$-cyle as a product of the remaining $k-1$ transpositions. The number of possibilities is therefore $k \cdot k^{k-2}=k^{k-1}$. So

$$
\left(1^{(2)}\right)^{k}=(k+1)^{k-1} \cdot 1^{(k+1)}+k^{k-1} \cdot 1^{(k, 2)}+\text { other terms. }
$$

Note that these two coefficients are coprime. Putting the two cases together, one gets, for a general element of $\operatorname{Sym}^{k} H^{2}\left(S^{[n]}, \mathbb{Z}\right), k=k_{0}+\ldots+k_{22}$

$$
\begin{aligned}
\left(1^{(2)}\right)^{k_{0}} \prod_{i=1}^{22}\left(\alpha_{i}^{(1)}\right)^{k_{i}}= & \left(k_{0}+1\right)^{k_{0}-1} \cdot 1^{\left(k_{0}+1\right)} \alpha_{1}^{\left(k_{1}\right)} \ldots \alpha_{22}^{\left(k_{22}\right)} \\
& +k_{0}^{k_{0}-1} \cdot 1^{\left(k_{0}, 2\right)} \alpha_{1}^{\left(k_{1}\right)} \ldots \alpha_{22}^{\left(k_{22}\right)}+\text { other terms. }
\end{aligned}
$$

One checks, that this is the only element of $\operatorname{Sym}^{k} H^{2}\left(S^{[n]}, \mathbb{Z}\right)$ having a non-zero coefficient at $1^{\left(k_{0}+1\right)} \alpha_{1}^{\left(k_{1}\right)} \ldots \alpha_{22}^{\left(k_{22}\right)}$ and $1^{\left(k_{0}, 2\right)} \alpha_{1}^{\left(k_{1}\right)} \ldots \alpha_{22}^{\left(k_{22}\right)}$. Now it is easy to show the existence of a complementary basis.

\section{Computational results}

We now give some results in low degrees, obtained by computing multiplication matrices with respect to the integral basis of $H^{*}\left(S^{[n]}, \mathbb{Z}\right)$. To get their cokernels, one has to reduce them to Smith normal form. Both results have been obtained using a computer.

REMARK 2.1. Denote the rank of $H^{k}\left(S^{[n]}, \mathbb{Z}\right)$ by $h^{k}\left(S^{[n]}\right)$.

- $h^{2}\left(S^{[n]}\right)=23$ for $n \geqslant 2$.

- $h^{4}\left(S^{[n]}\right)=276,299,300$ for $n=2,3, \geqslant 4$, respectively.

- $h^{6}\left(S^{[n]}\right)=23,2554,2852,2875,2876$ for $n=2,3,4,5, \geqslant 6$, respectively.

The algebra generated by classes of degree two is an interesting object to study. For cohomology with complex coefficients, Verbitsky has proved, in [12], that the cup product mapping from $\operatorname{Sym}^{k} H^{2}\left(S^{[n]}, \mathbb{C}\right)$ to $H^{2 k}\left(S^{[n]}, \mathbb{C}\right)$ is injective for $k \leqslant n$. Since there is no torsion, one concludes that this also holds for integral coefficients.

Proposition 2.2. We identify $\operatorname{Sym}^{2} H^{2}\left(S^{[n]}, \mathbb{Z}\right)$ with its image in $H^{4}\left(S^{[n]}, \mathbb{Z}\right)$ under the cup product mapping. Then

$$
\begin{aligned}
& \frac{H^{4}\left(S^{[2]}, \mathbb{Z}\right)}{\operatorname{Sym}^{2} H^{2}\left(S^{[2]}, \mathbb{Z}\right)} \cong\left(\frac{\mathbb{Z}}{2 \mathbb{Z}}\right)^{\oplus 23} \oplus \frac{\mathbb{Z}}{5 \mathbb{Z}}, \\
& \frac{H^{4}\left(S^{[3]}, \mathbb{Z}\right)}{\operatorname{Sym}^{2} H^{2}\left(S^{[3]}, \mathbb{Z}\right)} \cong \frac{\mathbb{Z}}{3 \mathbb{Z}} \oplus \mathbb{Z}^{\oplus 23}, \\
& \frac{H^{4}\left(S^{[n]}, \mathbb{Z}\right)}{\operatorname{Sym}^{2} H^{2}\left(S^{[n]}, \mathbb{Z}\right)} \cong \mathbb{Z}^{\oplus 24}, \quad \text { for } n \geqslant 4 .
\end{aligned}
$$

The 3-torsion part in (6) is generated by the integral class $1^{(3)}$. 
REMARK 2.3. The torsion in the case $n=2$ was also computed by Boissière et al. [1, Proposition 3] using similar techniques. To the best knowledge of the author, the result for $n=$ 3 is new. The freeness result for $n \geqslant 4$ has been proved already by Markman, [8, Theorem 1.10], using a completely different method.

Proposition 2.4. For triple products of $H^{2}\left(S^{[n]}, \mathbb{Z}\right)$,

$$
\frac{H^{6}\left(S^{[2]}, \mathbb{Z}\right)}{\operatorname{Sym}^{3} H^{2}\left(S^{[2]}, \mathbb{Z}\right)} \cong \frac{\mathbb{Z}}{2 \mathbb{Z}}
$$

The quotient is generated by the integral class $x^{(2)}$. Moreover,

$$
\begin{gathered}
\frac{H^{6}\left(S^{[3]}, \mathbb{Z}\right)}{\operatorname{Sym}^{3} H^{2}\left(S^{[3]}, \mathbb{Z}\right)} \cong\left(\frac{\mathbb{Z}}{2 \mathbb{Z}}\right)^{\oplus 230} \oplus\left(\frac{\mathbb{Z}}{36 \mathbb{Z}}\right)^{\oplus 22} \oplus \frac{\mathbb{Z}}{72 \mathbb{Z}} \oplus \mathbb{Z}^{\oplus 254}, \\
\frac{H^{6}\left(S^{[4]}, \mathbb{Z}\right)}{\operatorname{Sym}^{3} H^{2}\left(S^{[4]}, \mathbb{Z}\right)} \cong \frac{\mathbb{Z}}{2 \mathbb{Z}} \oplus \mathbb{Z}^{\oplus 552} .
\end{gathered}
$$

For $n \geqslant 5$, the quotient is free, by Theorem 1.17.

We study now cup products between classes of degree two and four. The case of $S^{[3]}$ is of particular interest.

Proposition 2.5. The cup product mapping : $H^{2}\left(S^{[n]}, \mathbb{Z}\right) \otimes H^{4}\left(S^{[n]}, \mathbb{Z}\right) \rightarrow H^{6}\left(S^{[n]}, \mathbb{Z}\right)$ is neither injective (unless $n=0$ ) nor surjective (unless $n \leqslant 2$ ).

$$
\begin{aligned}
& \frac{H^{6}\left(S^{[3]}, \mathbb{Z}\right)}{H^{2}\left(S^{[3]}, \mathbb{Z}\right) \smile H^{4}\left(S^{[3]}, \mathbb{Z}\right)} \cong\left(\frac{\mathbb{Z}}{3 \mathbb{Z}}\right)^{\oplus 22} \oplus \frac{\mathbb{Z}}{3 \mathbb{Z}}, \\
& \frac{H^{6}\left(S^{[4]}, \mathbb{Z}\right)}{H^{2}\left(S^{[4]}, \mathbb{Z}\right) \smile H^{4}\left(S^{[4]}, \mathbb{Z}\right)} \cong\left(\frac{\mathbb{Z}}{6 \mathbb{Z}}\right)^{\oplus 22} \oplus \frac{\mathbb{Z}}{108 \mathbb{Z}} \oplus \frac{\mathbb{Z}}{2 \mathbb{Z}}, \\
& \frac{H^{6}\left(S^{[5]}, \mathbb{Z}\right)}{H^{2}\left(S^{[5]}, \mathbb{Z}\right) \smile H^{4}\left(S^{[5]}, \mathbb{Z}\right)} \cong \mathbb{Z}^{\oplus 22} \oplus \mathbb{Z}, \\
& \frac{H^{6}\left(S^{[n]}, \mathbb{Z}\right)}{H^{2}\left(S^{[n]}, \mathbb{Z}\right) \smile H^{4}\left(S^{[n]}, \mathbb{Z}\right)} \cong \mathbb{Z}^{\oplus 22} \oplus \mathbb{Z} \oplus \mathbb{Z}, n \geqslant 6 .
\end{aligned}
$$

In each case, the first 22 factors of the quotient are generated by the integral classes

$$
\alpha_{i}^{(1,1,1)}-3 \cdot \alpha_{i}^{(2,1)}+3 \cdot \alpha_{i}^{(3)}+3 \cdot 1^{(2)} \alpha_{i}^{(1,1)}-6 \cdot 1^{(2)} \alpha_{i}^{(2)}+6 \cdot 1^{(2,2)} \alpha_{i}^{(1)}-3 \cdot 1^{(3)} \alpha_{i}^{(1)},
$$

for $i=1, \ldots, 22$. Now define an integral class

$$
\begin{aligned}
K:= & \sum_{i \neq j} B\left(\alpha_{i}, \alpha_{j}\right)\left[\alpha_{i}^{(1,1)} \alpha_{j}^{(1)}-2 \cdot \alpha_{i}^{(2)} \alpha_{j}^{(1)}+\frac{3}{2} \cdot 1^{(2)} \alpha_{i}^{(1)} \alpha_{j}^{(1)}\right] \\
& +\sum_{i} B\left(\alpha_{i}, \alpha_{i}\right)\left[\alpha_{i}^{(1,1,1)}-2 \cdot \alpha_{i}^{(2,1)}+\frac{3}{2} \cdot 1^{(2)} \alpha_{i}^{(1,1)}\right]+x^{(2)}-1^{(2)} x^{(1)}
\end{aligned}
$$

In the case $n=3$, the last factor of the quotient is generated by $K$.

In the case $n=4$, the class $1^{(4)}$ generates the 2 -torsion factor and $K-38 \cdot 1^{(4)}$ generates the 108-torsion factor.

In the case $n=5$, the last factor of the quotient is generated by $K-16 \cdot 1^{(4)}+21 \cdot 1^{(3,2)}$. 
If $n \geqslant 6$, the last two factors of the quotient are generated over the rationals by $K+$ $(4 / 3)(45-n) 1^{(2,2,2)}-(48-n) 1^{(3,2)}$ and $K+(1 / 2)(40-n) 1^{(2,2,2)}-(1 / 4)(48-n) 1^{(4)}$. Over $\mathbb{Z}$, one has to take appropriate multiples depending on $n$, such that the coefficients become integral numbers.

Proof. The last assertion for arbitrary $n$ follows from Corollary 1.13. First observe that for $\boldsymbol{\alpha}^{\boldsymbol{\lambda}} \in H^{2}, \boldsymbol{\alpha}^{\boldsymbol{\mu}} \in H^{4}, \boldsymbol{\alpha}^{\boldsymbol{\nu}} \in H^{6}$, we have $\|\boldsymbol{\lambda}\| \leqslant 2,\|\boldsymbol{\mu}\| \leqslant 4$ and $\|\boldsymbol{\nu}\| \geqslant 2$, according to Lemma 1.9. The coefficients of the cup product matrix are thus polynomials of degree at most $2+4-2=4$ and it suffices to compute only a finite number of instances for $n$. It turns out that the maximal degree is one and the cokernel of the multiplication map is given as stated.

In what follows, we compare some well-known facts about Hilbert schemes of points on K3 surfaces with our numerical calculations. This means that we have some tests that may justify the correctness of our computer program. We now state computational results for the middle cohomology group. Since $S^{[n]}$ is a projective variety of complex dimension $2 n$, Poincaré duality gives $H^{2 n}\left(S^{[n]}, \mathbb{Z}\right)$ the structure of a unimodular lattice.

Proposition 2.6. Let $L$ denote the unimodular lattice $H^{2 n}\left(S^{[n]}, \mathbb{Z}\right)$.

(1) For $n=2, L$ is an odd lattice of rank 276 and signature 156.

(2) For $n=3, L$ is an even lattice of rank 2554 and signature -1152.

(3) For $n=4, L$ is an odd lattice of rank 19298 and signature 7082.

For $n$ even, $L$ is always odd.

Proof. The numerical results come from an explicit calculation. For $n$ even, we always have the norm-one vector given by Example 1.16, so $L$ is odd. To obtain the signature, we could, equivalently, use Hirzebruch's signature theorem and compute the L-genus of $S^{[n]}$. For the signature, we need nothing but the Pontryagin numbers, which can be derived from the Chern numbers of $S^{[n]}$. These, in turn, are known by Ellingsrud et al. [3, Remark 5.5].

Another test is to compute the lattice structure of $H^{2}\left(S^{[2]}, \mathbb{Z}\right)$, with bilinear form given by $(a, b) \longmapsto \int\left(a \smile b \smile 1^{(2)} \smile 1^{(2)}\right)$. The signature of this lattice is 17 , as shown by Boissière et al. [1, Lemma 6.9].

\section{Appendix A. Source code}

We give the source code for our computer program. It is available online from https: //github.com/s--kapfer/HilbK3 and is also available as online supplementary material from the publisher's website. We used the language Haskell, compiled with the GHC software, version 7.6.3. We make use of two external packages: PERMUTATION and MEMoTRIE. The project is divided into four modules.

\section{A.1. How to use the code}

The main module is in the file HilbK3.hs, which can be opened by GHCI for interactive use. It provides an implementation of the ring structure of $A^{[n]}=H^{*}\left(S^{[n]}, \mathbb{Q}\right)$, for all $n \in \mathbb{N}$. It computes cup products in a reasonable time up to $n=8$. A product of Nakajima operators is represented by a pair consisting of a partition of length $k$ and a list of the same length, filled with indices for the basis elements of $H^{*}(S)$. For example, the class

$$
\mathfrak{q}_{3}\left(\alpha_{6}\right) \mathfrak{q}_{3}\left(\alpha_{7}\right) \mathfrak{q}_{2}(x) \mathfrak{q}_{1}\left(\alpha_{2}\right) \mathfrak{q}_{1}(1)^{2}|0\rangle
$$

in $H^{20}\left(S^{[11]}\right)$ is written as

*HilbK3> (PartLambda $[3,3,2,1,1,1],[6,7,23,2,0,0]$ ) : : AnBase 
Note that the classes $1 \in H^{0}(S)$ and $x \in H^{4}(S)$ have indices 0 and 23 in the code. The multiplication in $A^{[n]}$ is implemented by the method multAn.

The classes from Theorem 1.7 are represented in the same format, as shown in the following example. The multiplication in $H^{*}\left(S^{[n]}, \mathbb{Z}\right)$ of such classes is implemented by the method cupInt.

ExAmple A.1. We want to compute the results from Example 1.15. We only do one particular instance for every example, since the others are similar. By Corollary 1.13, it suffices to know the values for a finite number of $n$ to deduce the general case.

(1) We do the case $n=6, i=1$.

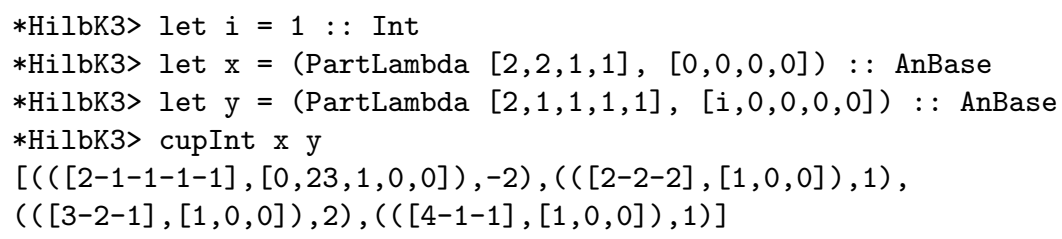

(2) We do the case $n=4, i=j=1$.

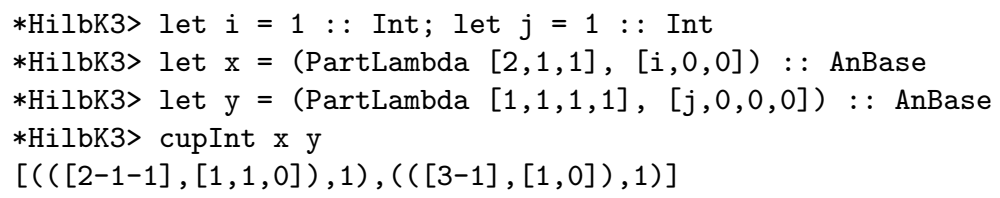

(3) We do the case $n=4$.

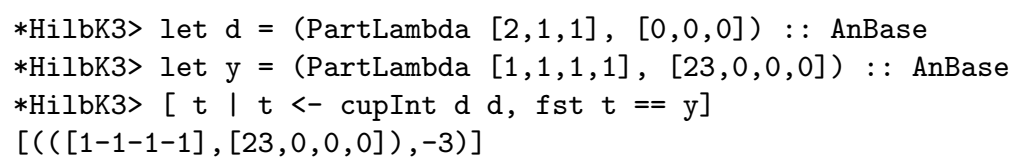

(4) We do the case $n=5$.

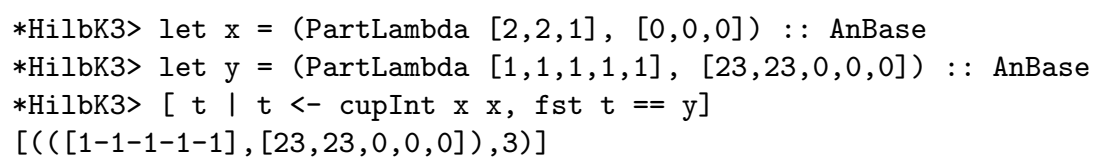

A.2. What the code does

The goal is to multiply two elements in $H^{*}\left(S^{[n]}, \mathbb{Z}\right)$. To do this, one has to execute the following steps.

(1) Compute the base change matrices $\psi_{\rho \nu}$ and $\psi_{\nu \rho}^{-1}$ between monomial and power sum symmetric functions.

(2) Provide a basis and the ring structure of $A=H^{*}(S, \mathbb{Z})$.

(3) Create a data structure for elements in $A^{[n]}$ and $A\left\{S_{n}\right\}$.

(4) Implement the multiplication in $A\left\{S_{n}\right\}$ : that is, the map $m_{\pi, \tau}$ from Definition 1.10.

(5) Implement the symmetrisation $A^{[n]}=A\left\{S_{n}\right\}^{S_{n}}$.

(6) Use the isomorphism from Theorem 1.11 to get the ring structure of $A^{[n]}$.

(7) Write an element in $H^{*}\left(S^{[n]}, \mathbb{Z}\right)$ as a linear combination of products of creation operators acting on the vacuum, using Theorem 1.7.

We now describe where to find these steps in the code.

(1) The $\psi_{\rho \nu}$ are computed by the function monomialPower in the module SymmetricFunctions.hs, using the theory from $[5, \S 3.7]$. The idea is to use the scalar product on the space of symmetric functions, so that the power sums become orthogonal: 
$\left(p_{\lambda}, p_{\mu}\right)=z_{\lambda} \delta_{\lambda \mu}$. The values for $\left(p_{\lambda}, m_{\mu}\right)$ are given by [5, Lemma 3.7.1], so we know how to get the matrix $\psi_{\nu \rho}^{-1}$. Since it is triangular with respect to some ordering of partitions, matrix inversion is easy.

(2) The ring structure of $H^{*}(S, \mathbb{Z})$ is stored in the module K3.hs. The only non-trivial multiplications are the products of two elements in $H^{2}(S, \mathbb{Z})$, where the intersection matrix is composed by the matrices for the hyperbolic and the $E_{8}$ lattice. The cup product and the adjoint comultiplication, from Definition 1.6, are implemented by the methods cup and cupAd.

(3) The data structures for basis elements of $A^{[n]}$ and $A\left\{S_{n}\right\}$ are given by AnBase and SnBase in the module HilbK3.hs. Linear combinations of basis elements are always stored as lists of pairs, each pair consisting of a basis element and a scalar factor.

(4) The function $m_{\pi, \tau}$, from Definition 1.10 , is computed by the method multSn. It contains the following substeps. First, the orbits of $\langle\pi, \tau\rangle$ are computed recursively by glueing together the orbits of $\pi$ with an orbit of $\tau$ if they have non-empty intersection. Second, the composition $\pi \tau$ is computed using a method from the external library Data.Permute. Third, the functions $f^{\pi,\langle\pi, \tau\rangle}$ and $f_{\langle\pi, \tau\rangle, \pi \tau}$ are computed using the (co-)products from K3.hs.

(5) The symmetrisation morphism is implemented by toSn. We do not know a better way to do this other than the naive approach which is summation over all elements in $S_{n}$.

(6) The multiplication in $A^{[n]}$ is carried out by the method multAn.

(7) The base change matrices between the canonical base of $A^{[n]}$ and the base of $H^{*}\left(S^{[n]}, \mathbb{Z}\right)$ are given by creaInt and intCrea. By composing multAn with these matrices, one gets the desired multiplication in $H^{*}\left(S^{[n]}, \mathbb{Z}\right)$, called cupInt.

\section{A.3. Module for cup product structure of K3 surfaces}

Here the hyperbolic and the $E_{8}$ lattice and the bilinear form on the cohomology of a K3 surface are defined. Furthermore, cup products and their adjoints are implemented.

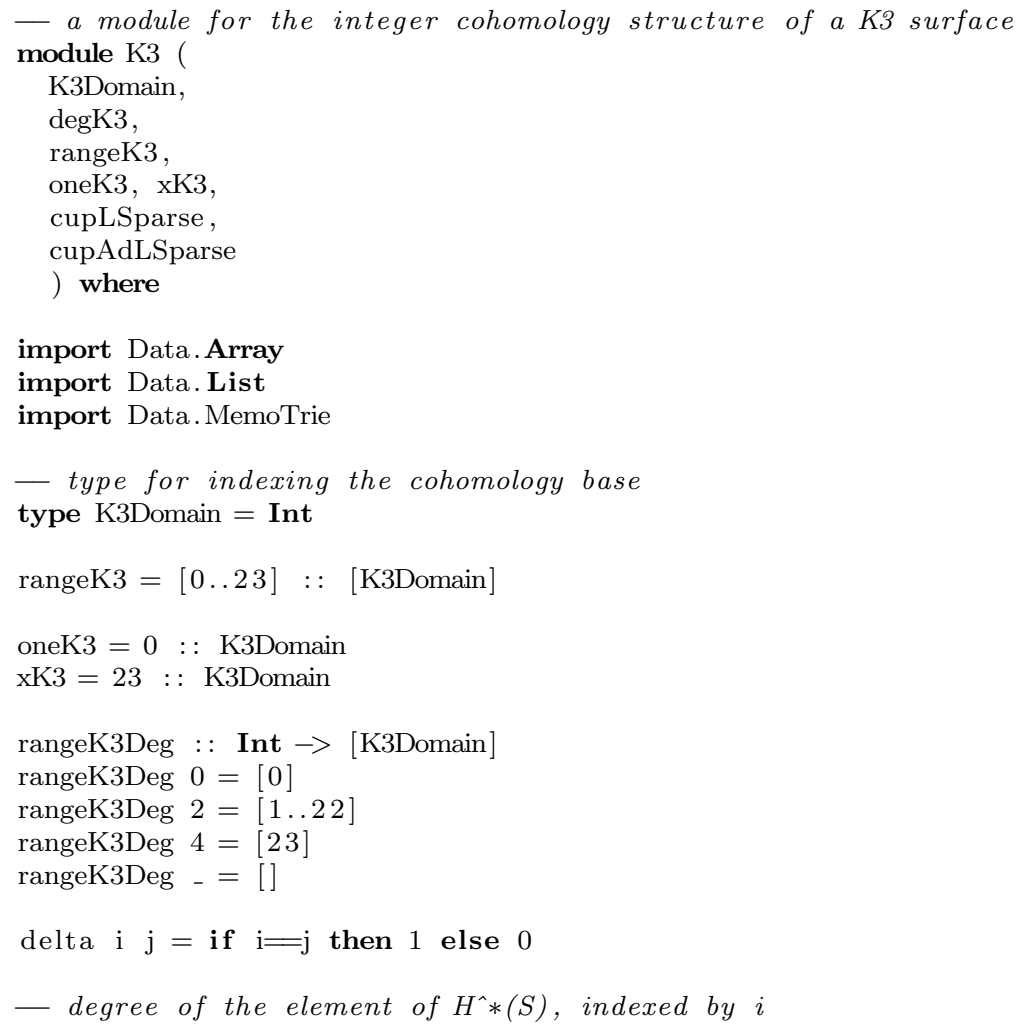




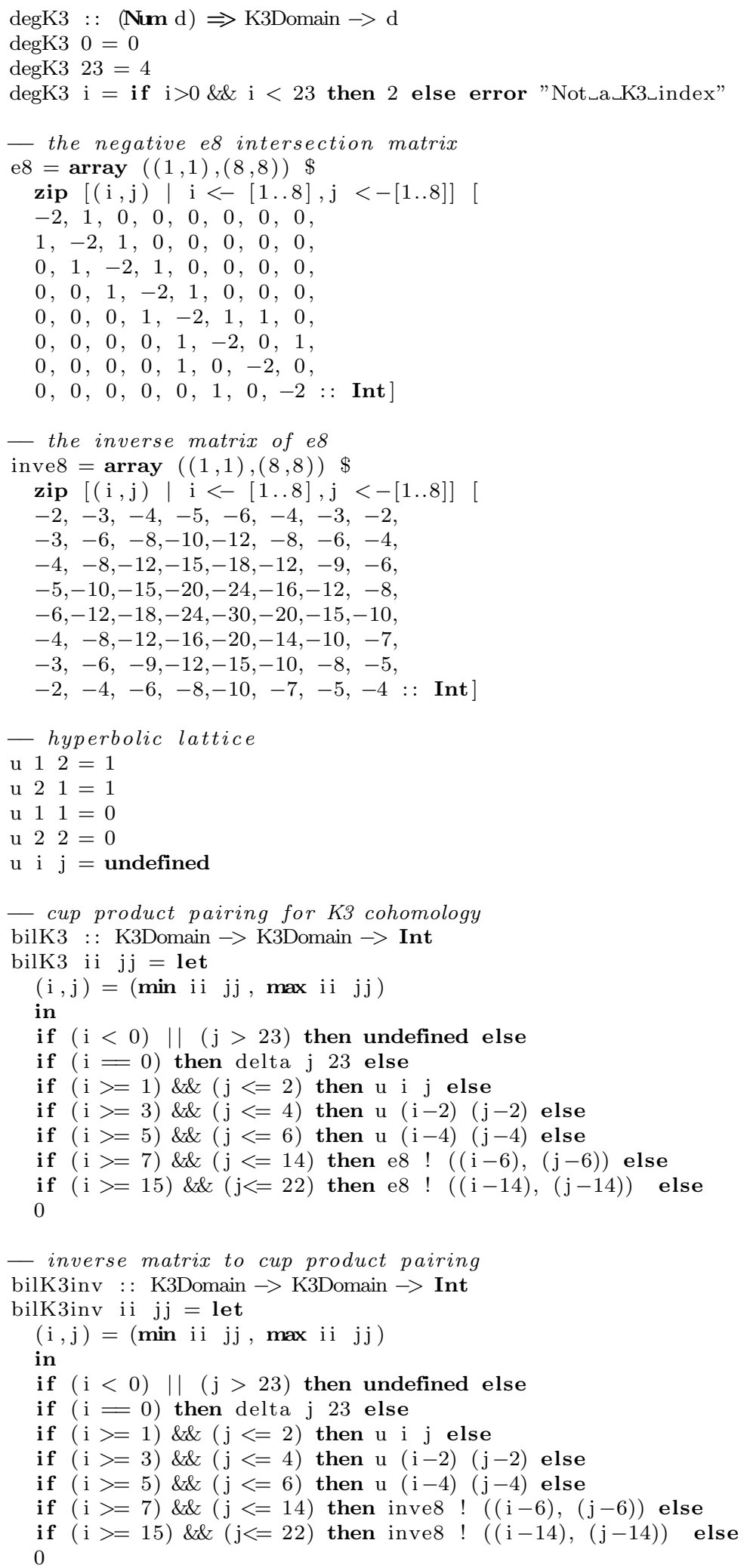



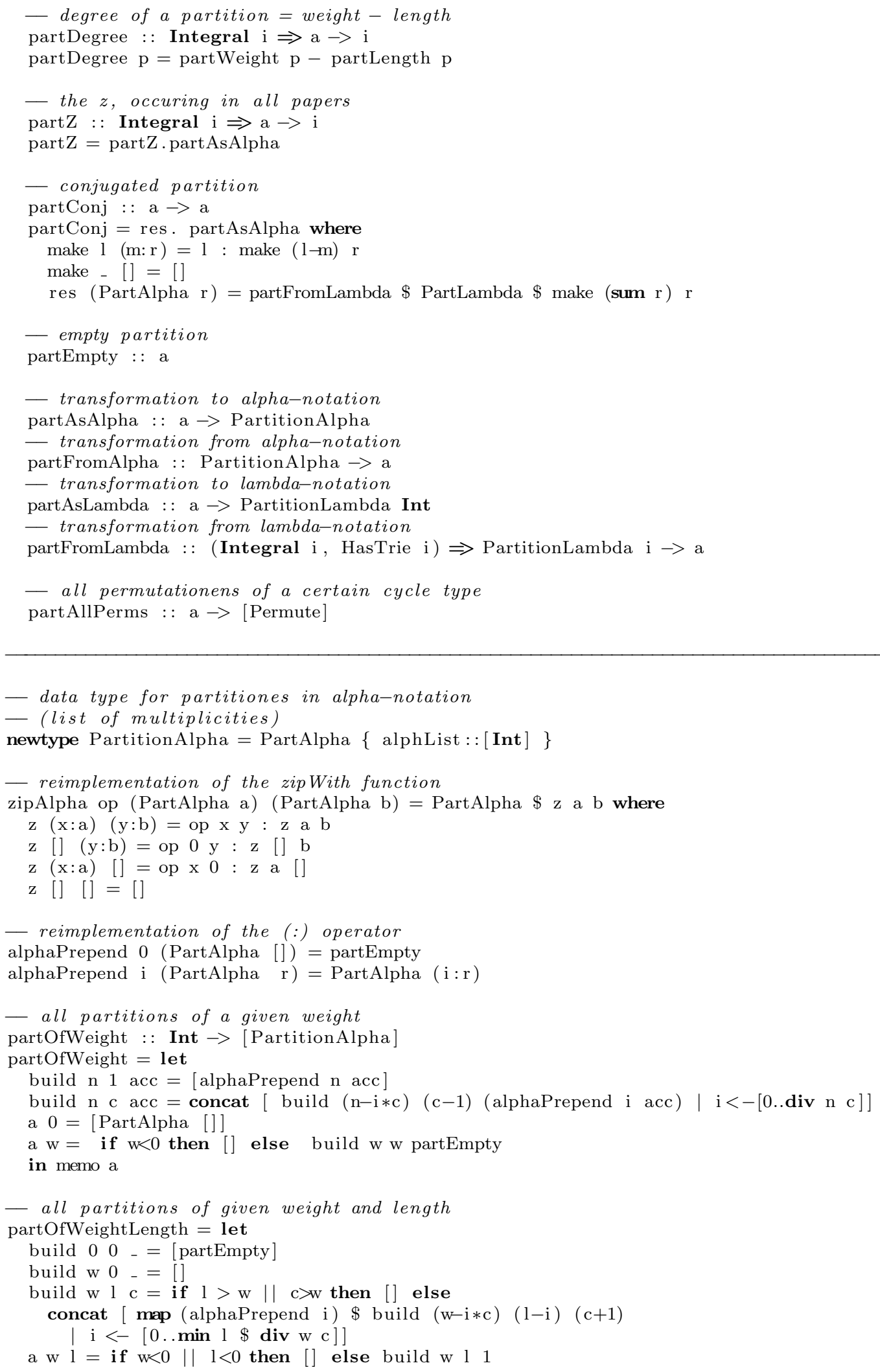
in memo2 a

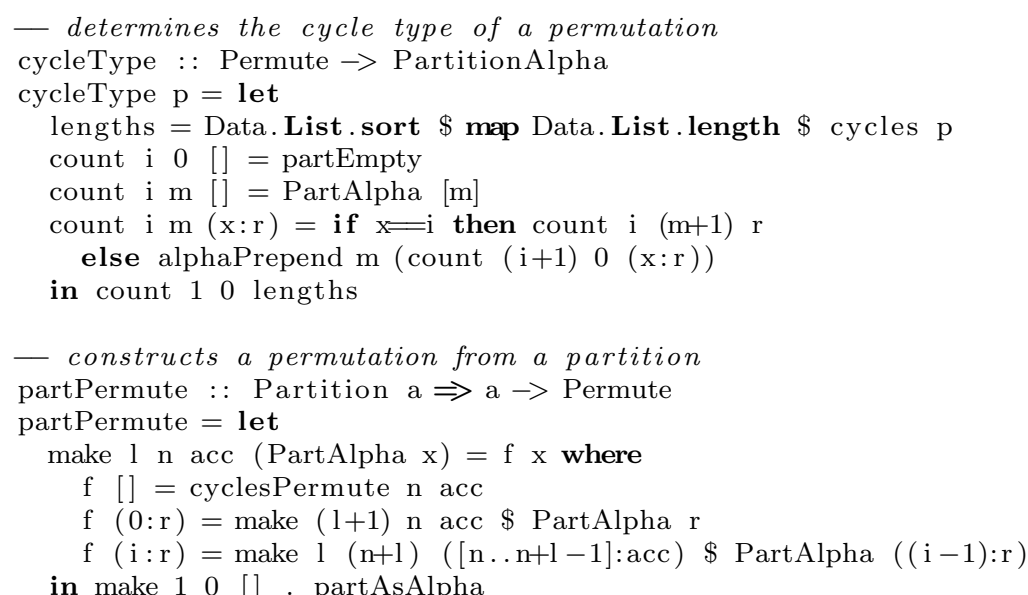

in make 10 [ 0 . partAsAlpha

instance Partition PartitionAlpha where

partWeight (PartAlpha $r)=$ fromIntegral $\$$ sum $\$$ zipWith $(*) r \quad[1 \ldots]$

partLength (PartAlpha $r$ ) = fromIntegral $\$$ sum $r$

partEmpty = PartAlpha []

partZ (PartAlpha 1$)=$ foldr $(*) 1 \$$

zipWith ( $\backslash \mathrm{a} i \rightarrow$ factorial $\left.a * \mathrm{i}^{\wedge} \mathrm{a}\right)$ (map fromIntegral l) [1..] where

factorial $\mathrm{n}=$ if $\mathrm{n}=0$ then 1 else $n *$ factorial $(n-1)$

partAsAlpha $=\mathbf{i d}$

partFromAlpha $=$ id

partAsLambda (PartAlpha 1) = PartLambda $\$$ reverse $\$$ f 1 l where

$\mathrm{f}$ i []$=[]$

$\mathrm{f}$ i $(0: \mathrm{r})=\mathrm{f}(\mathrm{i}+1) \mathrm{r}$

f i $(m: r)=i$ : f i $((m-1): r)$

partFromLambda = lambdaToAlpha

partAllPerms $=$ partAllPerms. partAsLambda

instance Eq PartitionAlpha where

PartAlpha $\mathrm{p}=$ PartAlpha $\mathrm{q}=$ findEq $\mathrm{p} \mathrm{q}$ where

findEq [] [] = True

findEq $(\mathrm{a}: \mathrm{p})(\mathrm{b}: \mathrm{q})=(\mathrm{a}=\mathrm{b})$ \&\& findEq $\mathrm{p} \mathrm{q}$

findEq [] $\mathrm{q}=$ isZero $\mathrm{q}$

findEq $\mathrm{p}[]=$ isZero $\mathrm{p}$

isZero = all $(==0)$

instance Ord PartitionAlpha where

compare a1 a2 = compare (partAsLambda a1) (partAsLambda a2)

instance Show PartitionAlpha where

show $\mathrm{p}=$ let

leftBracket $="(\mid "$

rightBracket $=" \mid) "$

rest [] = rightBracket

rest $[\mathrm{i}]=$ show $\mathrm{i}+$ rightBracket

rest $(\mathrm{i}: \mathrm{q})=$ show $\mathrm{i}++", "+$ rest $\mathrm{q}$

in leftBracket + rest (alphList $p$ )

instance HasTrie PartitionAlpha where

newtype PartitionAlpha $:->$ : $a=$ TrieType $\{$ unTrieType :: [Int $]:->$ : a $\}$

trie $\mathrm{f}=$ TrieType $\$$ trie $\$ \mathrm{f}$. PartAlpha

untrie $\mathrm{f}=$ untrie (unTrieType $\mathrm{f}$ ) . alphList

enumerate $\mathrm{f}=\operatorname{map}(\backslash(\mathrm{a}, \mathrm{b}) \rightarrow($ PartAlpha $\mathrm{a}, \mathrm{b})) \$$ enumerate (unTrieType $\mathrm{f}$ ) 


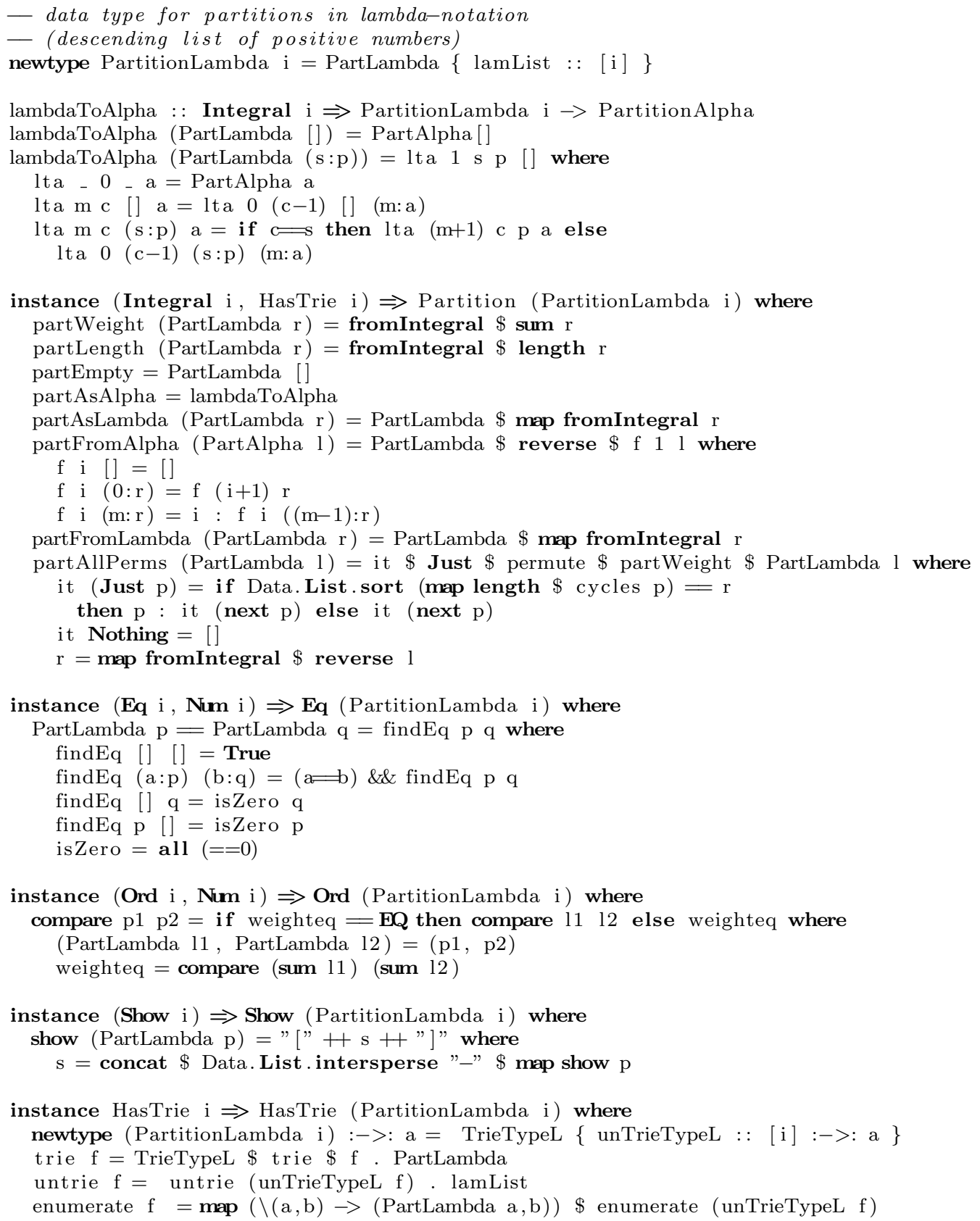

\section{A.5. Module for coefficients on symmetric functions}

This module provides only the base change matrices $\psi_{\lambda \mu}$ and $\psi_{\mu \lambda}^{-1}$, from Definition 1.2. 
) where

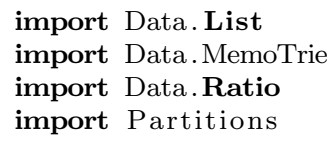

- binomial coefficients

choose $\mathrm{n} \mathrm{k}=\operatorname{ch} 1 \mathrm{n} \mathrm{k}$ where

$\operatorname{ch} 1=\operatorname{memo} 2 \mathrm{ch}$

ch $00=1$

ch $\mathrm{n} \mathrm{k}=$ if $\mathrm{n}<0 \quad \| \mathrm{k}<0$ then 0 else if $\mathrm{k}>\operatorname{div} \mathrm{n} 2+1$ then $\operatorname{ch} 1 \mathrm{n}(\mathrm{n}-\mathrm{k})$ else $\operatorname{ch} 1(n-1) k+\operatorname{ch} 1(n-1)(k-1)$

- multinomial coefficients

multinomial 0 [] $=1$

multinomial $\mathrm{n}[]=0$

multinomial $\mathrm{n}(\mathrm{k}: \mathrm{r})=$ choose $\mathrm{n} \mathrm{k} *$ multinomial $(\mathrm{n}-\mathrm{k}) \mathrm{r}$

- factorial function

factorial $0=1$

factorial $\mathrm{n}=\mathrm{n} *$ factorial $(\mathrm{n}-1)$

- http://uww.mat. univie.ac.at/ ^slc/wpapers/s68vortrag/ALCoursSf2.pdf , p. 48

- scalar product between monomial symmetric functions and power sums

monomialScalarPower moI poI $=(\mathrm{s} *$ partZ poI $)$ 'div' quo where

$\mathrm{mI}=$ partAsAlpha moI

$\mathrm{s}=\operatorname{sum}[\mathrm{a} *$ moebius $\mathrm{b} \mid(\mathrm{a}, \mathrm{b})<-$ finerPart $\mathrm{mI}$ (partAsLambda poI)]

quo $=$ product $[$ factorial $\mathrm{i} \mid$ let PartAlpha $1=\mathrm{mI}, \mathrm{i}<-1]$

nUnder 0[]$=[[]]$

nUnder $\mathrm{n}[]=[]$

nUnder $\mathrm{n}(\mathrm{r}:$ profile $)=$ concat $[\operatorname{map}(\mathrm{i}:) \$$ nUnder $(\mathrm{n}-\mathrm{i})$ profile $\mid \mathrm{i}<-[0$. min $\mathrm{n} r]]$

finerPart (PartAlpha a) (PartLambda 1$)=$ nub $\left[\left(\mathrm{a}^{\text {'div }}\right.\right.$ ' $^{\prime}$ sym sb, sb)

| $(\mathrm{a}, \mathrm{b})<-\mathrm{fp} 1$ a 1 , let $\mathrm{sb}=$ sort $\mathrm{b}]$ where

sym $=\begin{array}{lll}\mathrm{s} & 0 & {[1]}\end{array}$

$\mathrm{s} \mathrm{n}$ acc []$=$ factorial $\mathrm{n}$

$\mathrm{s} n$ acc $(\mathrm{a}: \mathrm{o})=$ if $\mathrm{a}=$ acc then $\mathrm{s}(\mathrm{n}+1)$ acc o else factorial $\mathrm{n} * \mathrm{~s} 1$ a $\mathrm{o}$

fp i []$\quad l=$ if all $(==0) 1$ then $[(1,[[] \mid x<-1])]$ else []

fp i $(0:$ ar $) \quad l=f p(i+1)$ ar $l$

$\mathrm{fp}$ i $(\mathrm{m}: \mathrm{ar}) \mathrm{l}=[(\mathrm{v} *$ multinomial $\mathrm{m}$ p, addprof $\mathrm{p}$ op $)$

| p <- nUnder m (map (flip div i) l),

$(\mathrm{v}, \mathrm{op})<-\mathrm{fp}(\mathrm{i}+1)$ ar (zipWith $(\backslash \mathrm{j} \mathrm{mm} \rightarrow \mathrm{j}-\mathrm{mm} * \mathrm{i}) \quad \mathrm{l} \mathrm{p})]$ where addprof $=\operatorname{zipWith~}(\backslash \mathrm{mm} l \rightarrow$ replicate $\mathrm{mm} \mathrm{i}++1)$

moebius $\mathrm{l}=$ product $\left[(-1)^{\wedge} \mathrm{c} *\right.$ factorial $\mathrm{c} \mid \mathrm{m}<-\mathrm{l}$, let $\mathrm{c}=$ length $\left.\mathrm{m}-1\right]$

- base change matrix from monomials to power sums

- no integer coefficients

- $m_{-} j=\operatorname{sum}\left[p_{-} i *\right.$ powerMonomial $i \quad j \mid i<-$ partitions]

powerMonomial : : (Partition a, Partition $\mathrm{b}) \stackrel{\mathrm{a} \rightarrow \mathrm{b} \rightarrow \text { Ratio Int }}{\Rightarrow}$

powerMonomial poI $\mathrm{moI}=$ monomialScalarPower moI poI $\%$ partZ poI

- base change matrix from power sums to monomials

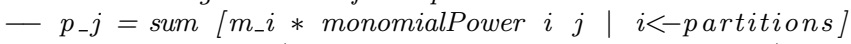

monomialPower : : (Partition a, Partition b, Num i) $\Rightarrow a \rightarrow b \rightarrow i$

monomialPower lambda $\mathrm{mu}=$ fromIntegral $\$$ numerator $\$$

memoizedMonomialPower (partAsLambda lambda) (partAsLambda mu)

memoizedMonomialPower $=$ memo2 mmpl where

mmpl $1 \mathrm{~m}=$ if partWeight $\mathrm{l}=$ partWeight $\mathrm{m}$ then mmp2 (partWeight $\mathrm{m}$ ) $1 \mathrm{~m}$ else 0

mmp2 $\mathrm{w} 1 \mathrm{~m}=$ invertLowerDiag (map partAsLambda $\$$ partOfWeight $\mathrm{w}$ ) powerMonomial $1 \mathrm{~m}$

- inversion of lower triangular matrix

invertLowerDiag vs $\mathrm{a}=\mathrm{ild}$ where

ild $=$ memo2 inv

delta $\mathrm{i} j=$ if $\mathrm{i}=\mathrm{j}$ then 1 else 0 
inv i j $\mid \mathrm{i}<\mathrm{j}=0$

otherwise $=(\operatorname{delta} \mathrm{i} j-\operatorname{sum}[\mathrm{a}$ i $\mathrm{k} * \mathrm{ild} \mathrm{k} \mathrm{j} \mid \mathrm{k}<-\mathrm{vs}, \mathrm{i}>\mathrm{k}, \mathrm{k}>=\mathrm{j}]) /$ a i i

A.6. Module implementing cup products for Hilbert schemes

This is our main module. We implement the algebraic model developed by Lehn and Sorger and the change of base due to Qin and Wang. The cup product on the Hilbert scheme is computed by the function cupInt.

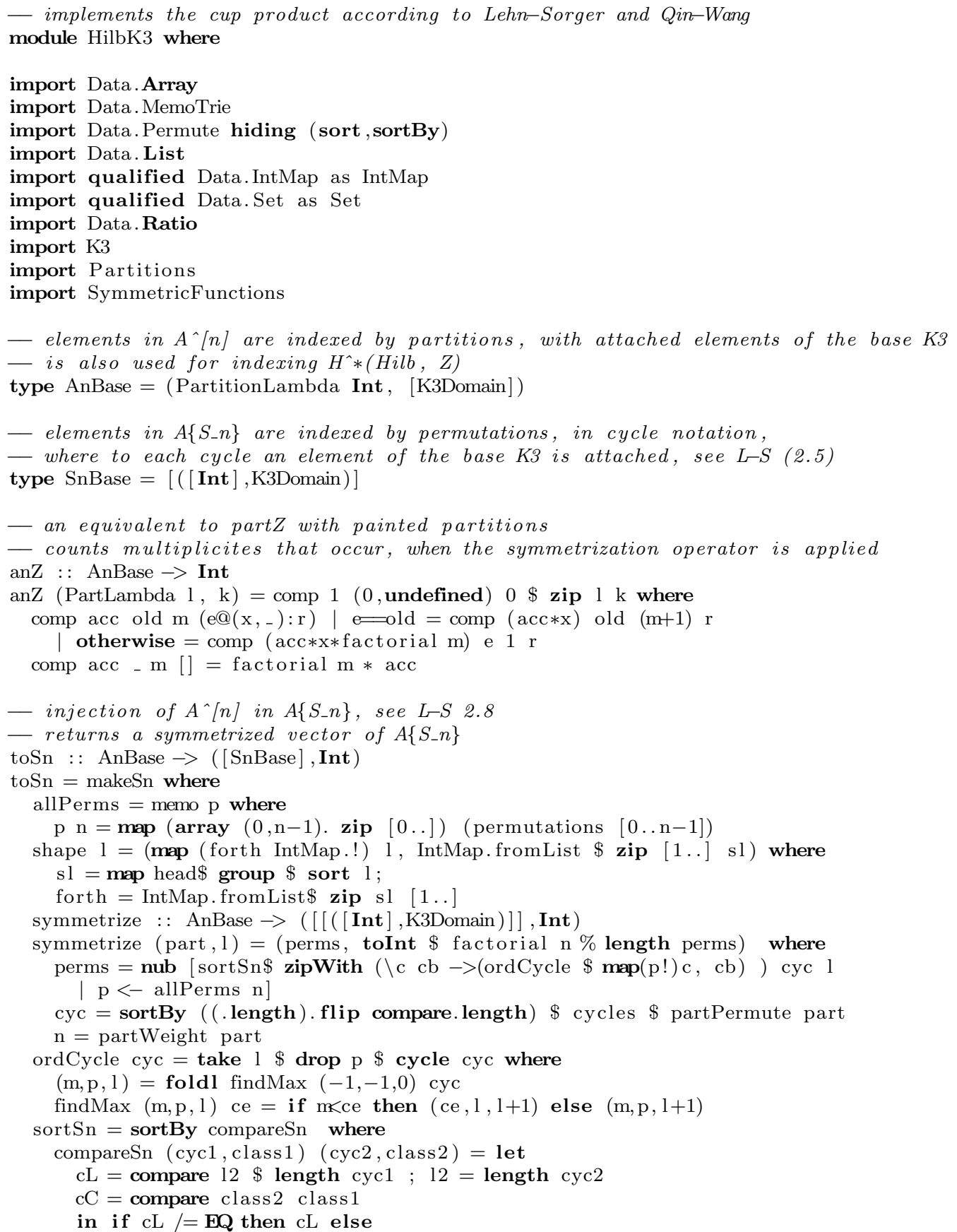


if $\mathrm{cC} /=\mathrm{EQ}$ then $\mathrm{cC}$ else compare $\mathrm{cyc} 2$ cyc1

mSym $=$ memo symmetrize

makeSn $($ part, 1$)=([[(\mathrm{z}$, im IntMap.$! \mathrm{k}) \mid(\mathrm{z}, \mathrm{k})<-$ op $] \mid$ op $<-$ res $], \mathrm{m}) \quad$ where

$($ repl, im $)=$ shape $l$

$($ res, $\mathrm{m})=$ mSym $($ part, $\mathrm{repl})$

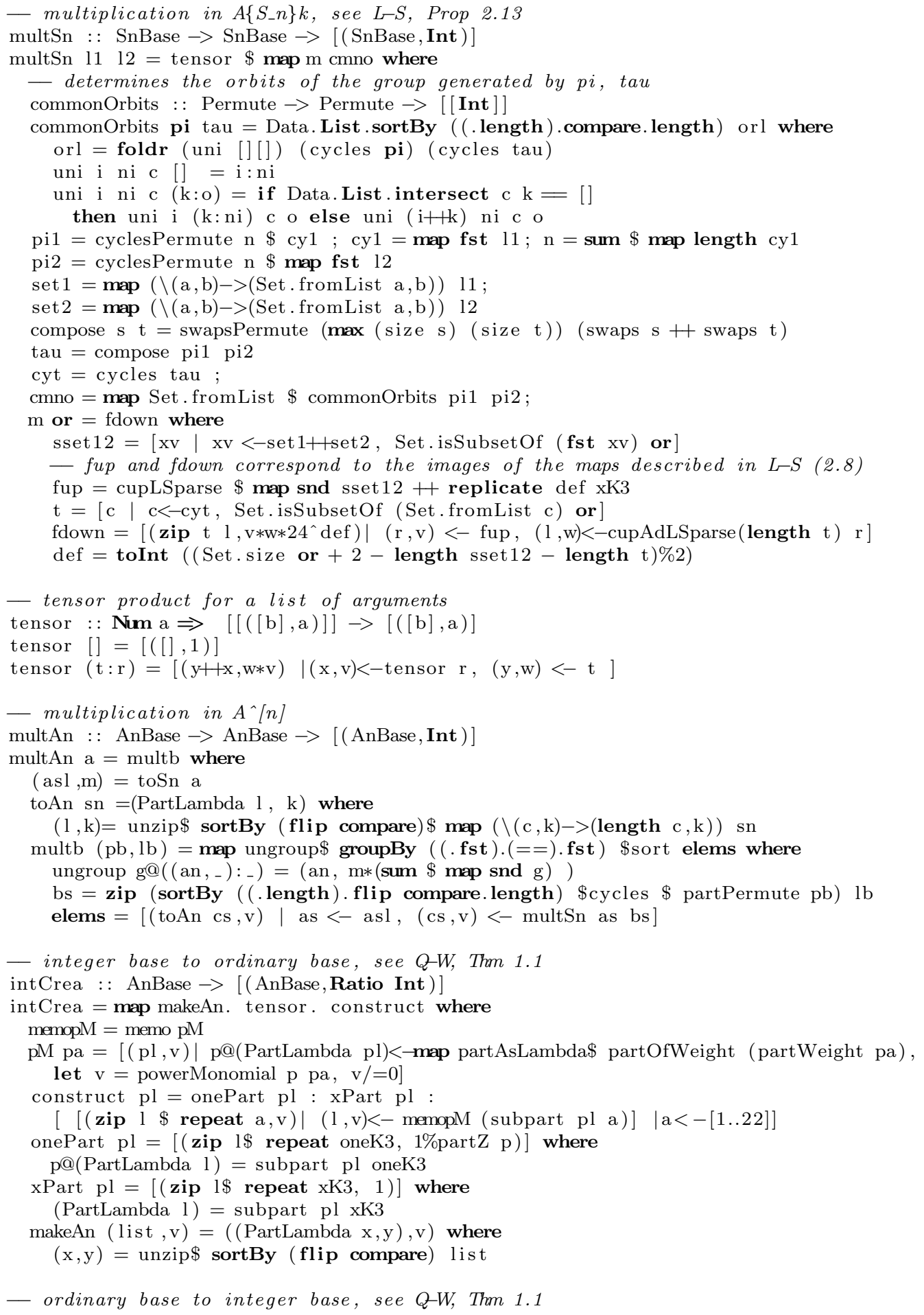




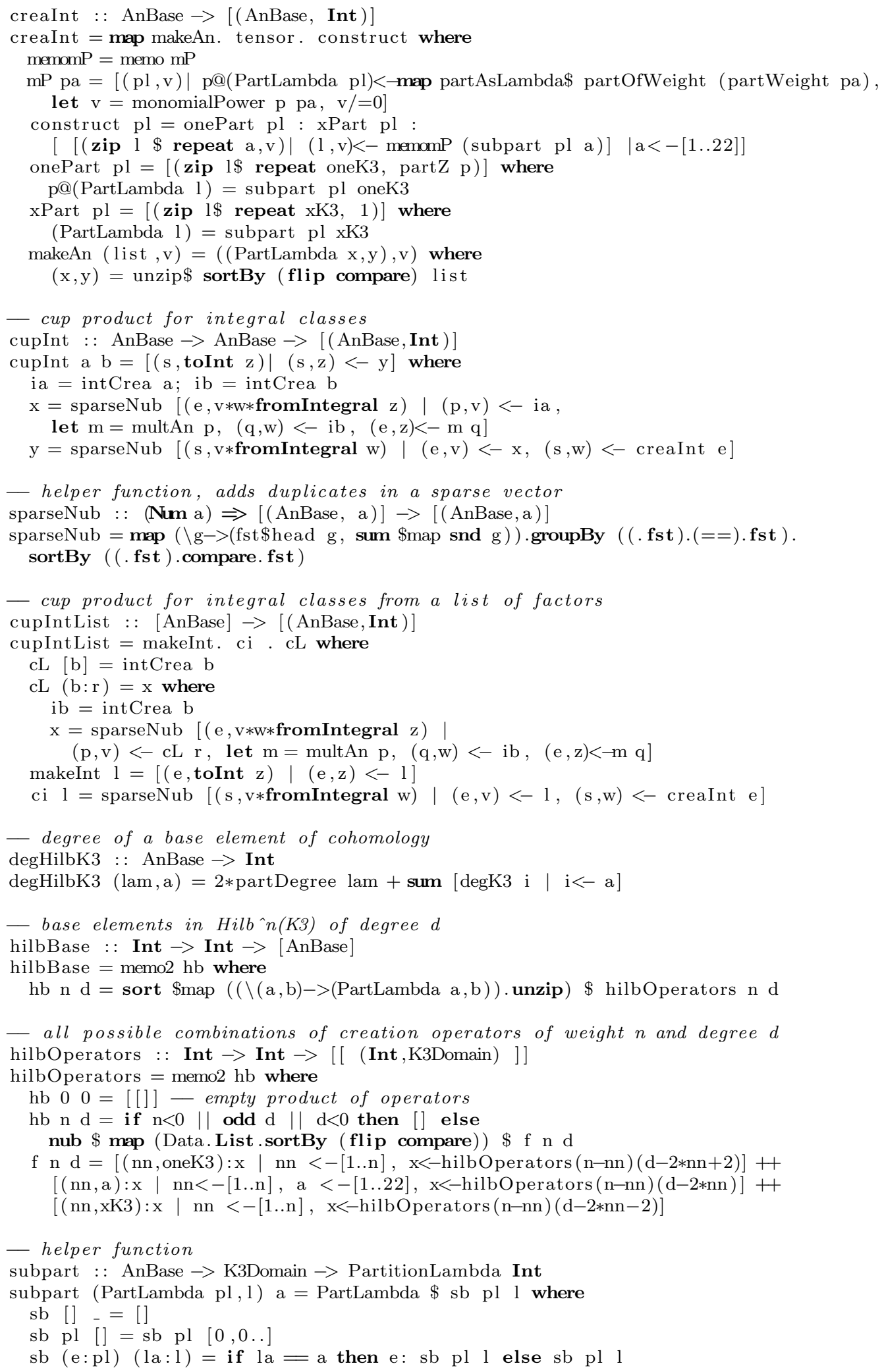




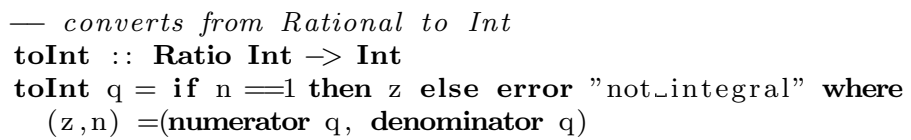

Acknowledgements. The author thanks Samuel Boissière and Marc Nieper-Wißkirchen for their supervision and many helpful comments. He also thanks Grégoire Menet for stimulating proposals and the Laboratoire de Mathématiques et Applications of the university of Poitiers for its hospitality. This work was partially supported by a DAAD grant.

\section{References}

1. S. Boissière, M. Nieper-WißKIrChen and A. SARti, 'Smith theory and irreducible holomorphic symplectic manifolds', J. Topol. 6 (2013) no. 2, 361-390.

2. J. DÉNES, 'The representation of a permutation as the product of a minimal number of transpositions, and its connection with the theory of graphs', Publ. Math. Inst. Hung. Acad. Sci. 4 (1959) 63-71.

3. G. Ellingsrud, L. Göttsche and M. Lehn, 'On the Cobordism class of the Hilbert scheme of a surface', J. Algebra. Geom. 10 (2001) 81-100.

4. J. Fogarty, 'Algebraic families on an algebraic surface', Amer. J. Math. 10 (1968) 511-521.

5. A. Lascoux, Symmetric functions, Notes of the course given at Nankai University (2001), http://www.mat.univie.ac.at/ slc/wpapers/s68vortrag/ALCoursSf2.pdf.

6. M. LeHn and C. Sorger, 'The cup product of Hilbert schemes for K3 surfaces', Invent. Math. 152 (2003) no. 2, 305-329.

7. E. MARKMAN, 'Integral generators for the cohomology ring of moduli spaces of sheaves over Poisson surfaces', Adv. Math. 208 (2007) no. 2, 622-646.

8. E. MARKMAN, 'Integral constraints on the monodromy group of the hyperKähler resolution of a symmetric product of a K3 surface', Internat. J. Math. 21 (2010) no. 2, 169-223.

9. J. Milnor and D. Husemöller, Symmetric bilinear forms, Ergebnisse der Mathematik und ihrer Grenzgebiete 73 (Springer, Berlin, 1973).

10. H. NAKajima, 'Heisenberg algebra and Hilbert schemes of points on projective surfaces', Ann. of Math. (2) 145 (1997) no. 2, 379-388.

11. Z. QIN and W. WANG, 'Integral operators and integral cohomology classes of Hilbert schemes', Math. Ann. 331 (2005) no. 3, 669-692.

12. M. Verbitsky, 'Cohomology of compact hyperkähler manifolds and its applications', Geom. Funct. Anal. 6 (1996) no. 4, 601-611.

\section{Simon Kapfer}

Laboratoire de Mathématiques et Applications

UMR CNRS 6086

Université de Poitiers

Téléport 2

Boulevard Marie et Pierre Curie

F-86962 Futuroscope Chasseneuil

France

simon.kapfer@math.univ-poitiers.fr 